\title{
Multilinguales
}

\section{La place de la grammaire énonciative dans les programmes et les manuels scolaires algériens du secondaire}

The Place of the Enunciative Grammar in the Programs and the Textbooks of the Algerian Secondary School

\section{Ahlem Idjet}

\section{OpenEdition Journals}

Édition électronique

URL : http://journals.openedition.org/multilinguales/938

DOI : 10.4000/multilinguales.938

ISSN : 2335-1853

\section{Éditeur}

Université Abderrahmane Mira - Bejaia

\section{Référence électronique}

Ahlem Idjet, « La place de la grammaire énonciative dans les programmes et les manuels scolaires algériens du secondaire », Multilinguales [En ligne], 6 | 2015, mis en ligne le 01 décembre 2015, consulté le 17 septembre 2019. URL : http://journals.openedition.org/multilinguales/938; DOI : 10.4000/multilinguales.938

Ce document a été généré automatiquement le 17 septembre 2019.

\section{(c)}

Multilinguales est mise à disposition selon les termes de la Licence Creative Commons Attribution Pas d'Utilisation Commerciale - Pas de Modification 4.0 International 


\title{
La place de la grammaire
} énonciative dans les programmes et les manuels scolaires algériens $\mathrm{du}$ secondaire

\author{
The Place of the Enunciative Grammar in the Programs and the Textbooks of the \\ Algerian Secondary School
}

\section{Ahlem Idjet}

1 Les programmes d'enseignement en application dans le secondaire ont pour origine la réforme élaborée dans le cadre d'un accord entre le Ministère algérien de l'Education Nationale et l'UNESCO (PARE, 2005)ํํㄹ au début des années 2000.

L'objectif de cette entreprise d'envergure est clairement fixé (PARE, op ; cit. : 8) :

En misant sur la refonte de la pédagogie, la rénovation des programmes et des manuels scolaires, la formation des encadreurs et l'élargissement de l'accès aux nouvelles technologies de linformation et de la communication, la réforme tente de répondre aux enjeux et aux défis d'assurer une plus grande pertinence de l'éducation dans une société en pleine mutation.

2 Cette réforme a conduit à une réorganisation totale du système scolaire et des programmes et à une redéfinition des compétences requises.

Nous nous proposons, dans ce travail, de développer une réflexion sur le traitement des contenus grammaticaux des nouveaux programmes de 2005 pour mettre au jour les fondements linguistiques de l'enseignement du FLE en général et de l'enseignement de la grammaire en particulier. Notre analyse porte donc sur les programmes et les manuels scolaires édités dans le cadre de la réforme scolaire.

Les programmes du secondaire sont des documents officiels élaborés par la commission nationale des programmes et publiés par l'office National des Publications Scolaires (O.N.P.S.). Ces documents sont destinés aux enseignants du cycle secondaire, et ont pour objectif de leur offrir une lecture fonctionnelle du nouveau programme qui 
dépasse le cadre de la classification typologique des textes en accordant une grande importance à la linguistique de l'énonciation.

\section{La place de la grammaire dans les manuels scolaires du secondaire}

3 Si l'on se réfère au préambule du Document d'accompagnement du programme de $3^{\text {ème }} \mathrm{AS}$, élaboré par la commission nationale des programmes, en septembre $2006^{2}$, le programme de 2005 se voulait en rupture avec l'enseignement traditionnel de la grammaire. Ses auteurs se sont tournés vers la pédagogie de la communication et la linguistique de l'énonciation :

Ce document est destiné aux professeurs du cycle secondaire.(..) Le nouveau programme poursuit des objectifs qui dépassent le cadre de la classification typologique des textes et accorde une grande importance à la linguistique de l'énonciation qui pose comme préalable qu'il faut distinguer ce qui est dit (contenu du texte) de la présence de l'énonciateur dans son propre discours.

Dans le nouveau programme de $1^{\text {ère }}$ Année Secondaire ( 1 ère $\mathrm{AS}^{3}$, par exemple, les rédacteurs, confirment l'orientation du programme général en insistant sur l'énonciation dès le préambule intitulé « Le cadre théorique »:

Le présent programme accorde une place plus importante à la linguistique de l'énonciation

(...). Ainsi, tout discours porte la marque de son énonciateur. En interrogeant ces traces d'énonciation, en se posant des questions sur le contexte d'énonciation (...), les modalités d'inscription du locuteur et de l'allocutaire dans l'objet - texte, l'opacité ou la transparence d'un texte, le degré d'objectivation du discours, - la focalisation (...),l'information contenue dans un texte sera appréhendée à un autre niveau de réflexion qui fera prendre conscience au fur et à mesure à l'apprenant qu'un locuteur est déterminé psychologiquement, idéologiquement, culturellement comme il l'est lui-même; que le référent dont parle le locuteur n'est pas le réel mais un réel filtré par lui; que le locuteur adresse toujours son message à un (ou des) allocutaire(s) ciblé(s).

Cette consigne est de nouveau confirmée dans le manuel scolaire de $1^{\text {ère }}$ A.S. :

La situation d'énonciation se définit par l'ensemble des facteurs qui déterminent la production d'un discours (nombre et personnalité des participants : présence de je/nous, tu/ vous, indices spatio-temporels liés au moment de l'énonciation "ici", "maintenant", marques de jugement. (Livre scolaire de $1^{\text {ère }} \mathrm{A} . \mathrm{S}$, projet 1 , séquence $1: 15$ )

Elle l'est également à la fin du même programme, dans la partie intitulée Glossaire qui consacre une entrée à Situation d'énonciation : « ensemble de facteurs qui déterminent la production d'un énoncé (nombre et personnalité des participants, cadre spatiotemporel, l'attitude de l'énonciateur par rapport au contenu de son énoncé) » (p. 51). Ainsi, le choix de la linguistique de l'énonciation est combiné, dans les nouveaux programmes, à celui de l'approche communicative; et la compétence de communication y est envisagée en ces termes :

(...) une compétence de communication n'est pas seulement la conjugaison de deux composantes (compétence linguistique et compétence textuelle). Pour être acquise, la compétence de communication doit intégrer d'autres compétences dont: la compétence sémiotico-sémantique (...), la compétence situationnelle et sociale (...), la compétence pragmatique (...). (Préambule, op. cit.)

Mais, en optant pour ce type d'approche, les manuels scolaires consacrent peu de place à l'exposé des savoirs proprement grammaticaux par rapport à des savoirs relatifs à la communication tels que les marques du dialogue ou l'argumentation. 
En effet, la prise en compte par les programmes de la situation de communication (programme de $1^{\text {ère }}$ A.S.) et des éléments extralinguistiques, du discours rapporté

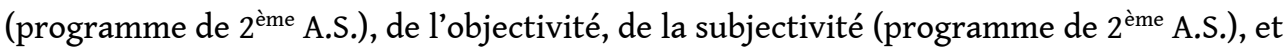
de la modalisation (programmes de $2^{\text {ème }}$ A.S. et $3^{\text {ème }}$ A.S.), relève de la grammaire énonciative.

Parmi les activités de langue proposées, nous trouvons peu de leçons qui portent sur l'aspect grammatical de la langue, et les contenus grammaticaux prescrits sont toujours rattachés à des types particuliers de discours : l'étude des valeurs de l'imparfait dans la description, l'emploi de l'impératif dans le texte exhortatif, la tournure impersonnelle dans le texte expositif, la caractérisation dans le texte descriptif, etc.

Les manuels scolaires conçus selon l'approche communicative proposent essentiellement l'enseignement des actes de parole tels qu'argumenter, exposer, relater, dialoguer, convaincre, ... D'ailleurs, le profil d'entrée en $1^{\text {ère }} \mathrm{AS}^{4}$ et l'un des trois "objets d'étude» du programme de $3^{\text {ème }} \mathrm{AS}^{5}$ reposent en grande partie sur l'argumentation.

On fait donc appel, du début à la fin du cycle secondaire, à la linguistique de la parole ou linguistique de l'énonciation, au détriment de la linguistique de la langue.

À ce niveau, la grammaire est une grammaire notionnelle-fonctionnelle qui vise le renforcement de la compétence discursive de l'élève. En effet, s'inscrivant dans le cadre de l'approche communicative,

Une approche notionnelle de la grammaire ne tente rien d'autre que de présenter - et parfois d'expliquer - les faits grammaticaux de manière à ce que se construisent progressivement, dans l'esprit des apprenants, de nouvelles représentations de sens, elle tente de créer des références notionnelles. Ces références pour se construire, ne peuvent partir que de notions simples, claires, partagées par tous ou qu'il est possible de faire partager. (...) L'approche notionnelle de la grammaire s'applique en réalité à un assez petit nombre d'éléments. (...) Il s'agit de mémoriser la chaîne syntaxique et d'apprendre le lexique. C'est ce qui se passe dans un premier temps. Peu d'attention est portée par les apprenants à la grammaire en tant que telle. (Courtillon, $1985: 36$ )

Cette grammaire permet de décrire les différentes valeurs et formes que prennent les faits syntaxiques dans les actes de langage et les situations de communication. Elle repose sur l'hypothèse selon laquelle l'acquisition des structures grammaticales favorise l'acquisition de l'acte de parole et donc de la compétence de communication. Nous pouvons citer, à titre d'exemple, l'enseignement de la tournure impersonnelle dans le manuel de $2^{\text {ème } A S}$ : «L'emploi de la forme impersonnelle dans le texte explicatif permet d'effacer la présence et les marques de l'énonciateur » (p.81). La notion est conçue dans sa fonction discursive dans le contexte du texte explicatif et dans d'autres.

Cette grammaire se prête aux approches communicatives étant donné qu'elle est fondée sur la situation de communication, ce qui justifie le contenu des programmes du cycle secondaire qui, tout en s'inspirant de son modèle théorique (décrire et identifier les traces de l'acte d'utilisation de la langue dans le produit: l'énoncé), propose principalement des actes de paroles.

Mais la notion d'énonciation, récemment introduite en didactique de la langue dans les nouveaux programmes du secondaire, avec la réforme du système éducatif algérien, ne se laisse pas aisément appréhender parce qu'elle couvre un ensemble vaste et hétérogène de faits de langue.

Il est difficile en effet de traiter, d'un point de vue didactique, la linguistique énonciative dans son intégralité et sa complexité ; l'énonciation étant composée de 
notions ayant des relations avec d'autres notions elles-mêmes très vastes comme celles de la temporalité, de la modalisation et de l'argumentation.

Nous tenterons dans la section suivante d'analyser le discours des manuels scolaires publiés à partir de la rentrée scolaire 20042005 dans le cadre de la réforme scolaire et de repérer les traces discursives de l'énonciation, afin de faire émerger la ou les théories de l'énonciation visées.

\section{L'énonciation dans les programmes scolaires}

6 Comme signalé plus haut, la Commission Nationale insiste sur la place réservée à la linguistique de l'énonciation dont elle rappelle la définition, dans le Document d'accompagnement du programme de $3^{\text {ème }} A S$ de septembre 2006 (cf. supra).

A ce niveau, nous notons l'influence de la contribution de Kerbrat-Orecchioni au développement de la linguistique énonciative. En effet, la définition retenue dans ce document prend en charge tous les paramètres qu'elle a introduits pour enrichir le schéma de base de la communication: les compétences culturelles, idéologiques, paralinguistiques et les déterminations psychologiques :

Il est parfois impossible de décrire adéquatement les comportements verbaux sans tenir compte de leur environnement non-verbal; on ne peut étudier le sens sans envisager son référent; on ne peut décrire un message sans tenir compte de son contexte, des effets qu'il prétend obtenir. (Kerbrat-Orecchioni, 1988:68)

Il s'agit pour les auteurs du document d'emprunter à la grammaire de l'énonciation ses principes théoriques (définitions et notions) et ses méthodes pratiques (dégager la situation d'énonciation, relever les marques de l'énonciation, présence/absence de l'énonciateur dans son discours, ...) pour enseigner la grammaire aux élèves.

Nous avons analysé les programmes afin de repérer les traces discursives de l'énonciation qui apparaissent non seulement au niveau du "cadre théorique » des programmes mais aussi au niveau des « contenus " proposés pour les trois paliers ainsi présentés dans Le Document d'accompagnement :

Les contenus ont été sélectionnés, et leur progression sur l'année a été établie sur la base : de la prise en compte des types d'ancrage énonciatif (...), du croisement entre formes discursives et intentions communicatives, du passage d'objets d'étude dans lesquels les traces de l'énonciation sont perceptibles à des discours dans lesquels il s'agit de découvrir l'instance discursive, de la prise en compte des manifestations tant orales qu'écrites de la langue dans les différents supports que l'apprenant peut rencontrer dans son environnement extra scolaire (...). (op. cit. : 36)

Ces critères de sélection des contenus déterminent le traitement détaillé des objets d'étude. Ainsi, nous avons pu relever des traces de la linguistique énonciative à différents niveaux: notions clés (référence situationnelle, référence cotextuelle, description objective, description subjective), niveau discursif, niveau textuel et niveau phrastique.

Dans les trois programmes du secondaire, la linguistique de l'énonciation permet de mettre en relation des éléments linguistiques avec des paramètres situationnels: effacement du locuteur, la référence situationnelle, lexique appréciatif/dépréciatif (Programme de $1^{\text {ère }}$ A.S), modalités appréciatives traduisant l'opinion et le jugement de

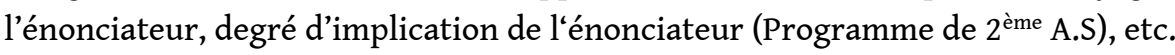




\section{L'énonciation dans les manuels scolaires}

7 Il nous faut relever, cependant, que, dans les manuels, l'énonciation est définie de manière abstraite et en quelques mots dans le Glossaire qui accompagne les programmes (cf. supra).

Le plus souvent, la notion apparaît dans les parties consacrées à la situation d'énonciation, elle-même décrite en reprenant fidèlement les instructions officielles :

La situation d'énonciation se définit par l'ensemble des facteurs qui déterminent la production d'un discours (nombre et personnalité des participants : présence de je/tu, nous/ vous, indices spatio-temporels liés au moment de l'énonciation ici, maintenant, marques de jugement)». (Manuel de $1^{\text {ère }}$ A.S : 15)

Nous avons pu constater également que plusieurs textes sont étudiés d'un point de vue énonciatif. En effet, les questions posées dans la partie «lecture analytique » mettent l'accent sur la situation d'énonciation et le cadre énonciatif. On insiste sur les marques de l'énonciation, les éléments qui traduisent la présence du locuteur, le nombre d'interlocuteurs et les marques qui renvoient à chacun d'eux, la relation entre situation de communication et situation d'énonciation, l'implication de l'auteur dans son texte. En voici un échantillon :

\section{Manuel de $1^{\text {ère }}$ AS}

«Comparez les deux discours au niveau du nombre de traces de l'énonciation et de la manifestation du destinataire » (p. 15),

« Relevez les marques de l'énonciation dans chacun des textes» (p. 46),

« Retrouvez les marques de l'énonciation au niveau lexical et syntaxique» (p. 139);

\section{Manuel de $2^{\text {ème }} \mathrm{AS}$}

«La présence de l'énonciateur et celle du destinataire sont-elles marquées?» (p. 29),

«A quelle situation d'énonciation renvoient ces propos directs?» (p. 186),

«Qu'exprime ce temps par rapport au moment de l'énonciation?» (p. 186).

Nous pouvons dire que le point de vue énonciatif est privilégié dans l'analyse des textes, comme dans les deux premiers projets du manuel scolaire de $1{ }^{\text {ère }}$ A.S. :

- projet 1 : Réaliser une campagne d'information à l'intention des élèves du lycée,

- projet 2 : Rédiger une lettre ouverte à une autorité compétente pour la sensibiliser à un problème et lui proposer des solutions.

On cherche à faire analyser la situation d'énonciation à travers une mise en relation des éléments linguistiques et des paramètres situationnels et à dégager les indices qui renvoient aux conditions de production du message et à l'inscription du sujet parlant dans son propre discours.

$\mathrm{Au}$ niveau des exercices proposés en fin de projet, les élèves sont mis au contact d'un métalangage emprunté à la grammaire énonciative. Citons à titre d'exemple,

- l'exercice $n^{\circ} 3$ (Manuel de $3^{\text {ème }}$ A.S. : 219) : « relevez les indices temporels et précisez si leur repère est le moment de l'énonciation ou un autre élément de la phrase ";

- l'exercice $n^{\circ} 4$ (Manuel de $3^{\text {ème }}$ A.S. : 62) : «dans les phrases suivantes relevez les indices temporels et dites s'ils ont un repère fixe (si le repère est le moment de l'énonciation ou un autre élément de la phrase)». 
En effet, parmi les formes linguistiques révélatrices de l'expérience énonciative, celles qui expriment le temps sont les plus évidentes. La localisation temporelle en français s'effectue essentiellement grâce aux formes temporelles de la conjugaison verbale, aux adverbes et locutions adverbiales qui exploitent un système de repérage déictique; le moment de l'énonciation représentant le repère à partir duquel on détermine les deux périodes, passée et future.

- Exercice $\mathrm{n}^{\circ} 6$ (Manuel de $3^{\text {ème }}$ A.S. : 62): les élèves doivent "ajoute(r) une modalité de l'incertitude dans chaque énoncé, c'est-à-dire une réserve de l'énonciation par rapport à l'énoncé». On leur explique qu'il s'agit d'une réserve de l'énonciation par rapport à l'énoncé, autrement dit une opération qui permet à l'énonciateur de prendre position par rapport à son énoncé et de s'y impliquer plus ou moins explicitement.

- Exercice $\mathrm{n}^{\circ} 1$ (Manuel de $3^{\text {ème }}$ A.S. : 218) : on propose aux élèves de «conjugue( $\mathrm{r}$ ) les verbes entre parenthèses tout en sachant que les deux premiers extraits sont coupés de la situation d'énonciation et que le troisième est une intervention du locuteur ». En effet, la linguistique de l'énonciation opère une distinction entre les énoncés dont les repérages se rapportent à la situation d'énonciation, énoncés ancrés, et ceux dont les repérages se rapportent à un terme de l'énoncé, énoncés coupés de la situation d'énonciation.

Plusieurs notions grammaticales (la forme impersonnelle, le discours rapporté, la voix passive, les temps verbaux, les discours direct et indirect, etc.) sont donc mises explicitement en perspective par rapport à la notion d'énonciation.

L'argumentation est également appréhendée d'un point de vue énonciatif : l'étude des textes argumentatifs en 3ème année secondaire permet de mettre l'accent sur les stratégies d'appel de l'auteur-énonciateur qui visent à faire agir ou réagir les lecteurs ainsi que sur celles des textes polémiques. Ce type de texte, dans lequel l'auteur appuie ou réfute une proposition, donne une importance à une énonciation qui évite la neutralité de l'expression. L'auteur utilise un style oratoire destiné à convaincre et à agir sur le destinataire ainsi que des injonctions qui interpellent le lecteur et sollicitent une réaction de sa part. Selon le programme de $3^{\text {ème }}$ A.S., l'auteur d'un texte argumentatif défend une prise de position, un point de vue, il s'oppose implicitement ou explicitement à ceux qui défendent le point de vue inverse.

Les élèves sont ainsi confrontés à une nouvelle terminologie empruntée à la linguistique de l'énonciation.

Quant à la notion de modalisation, définie comme « la forme linguistique d'un jugement intellectuel, d'un jugement affectif ou d'une volonté qu'un sujet parlant énonce à propos d'une perception ou d'une représentation de son esprit» (Bally, 1942:3), ses relations avec l'énonciation se situent essentiellement au niveau de la terminologie utilisée: " énonciateur » « destinataire » et " énoncé ».

Ainsi, on peut lire, dans le Manuel de $2^{\text {ème }}$ A.S. (2005 : 78), à la leçon sur les modalités appréciatives: "celui qui parle ou écrit s'exprime sur un fait en portant une appréciation (positive ou négative). Ce jugement repose sur le sentiment et non sur la raison ».

Dans ce passage, on décèle les traces de la définition de Bally (cf. supra).

Dans le même manuel, au niveau de la leçon sur l'appréciation et le jugement et dont l'objectif est de faire acquérir les modalisateurs (Manuel de $2^{\text {ème }}$ A.S. : 80), on explique aux élèves qu'

on informe son destinataire de quelque chose dont on est sûr, pour ce faire on utilise des modalisateurs...on est aussi amené à évaluer, à apprécier, à juger. Appréciation négative ou positive, il s'agit d'un jugement par le biais duquel on exprime un point de vue. 
On enseigne donc aux élèves que la modalisation est un moyen pour l'énonciateur de prendre position par rapport à son énoncé et/ou à son co-énonciateur; d'où sa superposition avec la notion de subjectivité dans son opposition à l'objectivité :

L'objectivité, c'est la description fidèle d'une réalité. Celui qui produit l'énoncé essaie d'effacer toute marque de sa présence dans cet énoncé. La subjectivité c'est la description personnelle d'une réalité. Celui qui produit l'énoncé s'implique directement ou indirectement. (Manuel de $2^{\text {ème }}$ A.S. : 146).

Or, les chercheurs, comme Elalouf et Kerinec (1999), Boch et Grossmann (2007) ${ }^{6}$ ont déjà traité de la confusion que cette synonymie entre modalisation et subjectivité entraînait chez les élèves :

L'approche de la modalisation dans les manuels met au jour la difficulté à cerner la notion et à en proposer une définition claire et utile pour les élèves. (...) Si on l'entend classiquement, et c'est le cas dans les manuels, comme le processus par lequel le sujet de l'énonciation manifeste son attitude à l'égard de son discours, alors la modalisation - synonyme de subjectivité - est partout: "la présence de l'énonciateur est consubstantielle à l'énoncé. (Elalouf et Kerinec, 1999 : 100)

Elalaouf et Kerinec, cités par Boch et Grosmann, mettent l'accent sur une autre difficulté de la notion de modalisation :

M. L. Elalouf et M. Kerinec (1999) avaient déjà bien identifié en quoi elle pose problème dans le cadre de l'enseignement; la variabilité à l'ouvre dans la définition qu'en donnent les ouvrages de référence serait l'une des raisons du caractère insaisissable de la notion, plus ou moins extensible. (idem).

L'un des effets de cette «confusion » relevé par ces chercheurs est celui « des listes démesurées, soumises à une forte variabilité dans les manuels des marques de modalisation» (idem).

Certes, ils analysent un contexte différent de celui de l'Algérie, mais force est de constater que l'enseignement de la modalisation se fait sur le même principe de sa synonymie avec la subjectivité et provoque les mêmes effets au niveau du relevé pléthorique des marques, et débouche sur le même résultat : la grande difficulté de son acquisition par les élèves.

La page 146 du manuel de $2^{\text {ème }}$ A.S., par exemple, contient une liste des marques explicites de la subjectivité auxquelles l'enseignant doit sensibiliser les élèves: des verbes de sentiment, de perception, d'opinion, de jugement, de perception, etc.; des adjectifs dans des constructions personnelles/impersonnelles, des adverbes et locutions adverbiales (jugement de vérité, jugement de réalité, autres...), etc.

Nous constatons que la liste des réalisateurs linguistiques de la modalité ne peut être fixe et que ceux-ci se manifestent dans des catégories différentes, dans des structures différentes, à des niveaux différents,

De plus, il existe autant de listes que de modalités (cf. p. 78/79/80 du même manuel). Cette confusion, qui entrave l'appropriation des outils de modalisation par les élèves, est renforcée par l'opposition "énoncé subjectif»/《énoncé objectif » assimilé à un «énoncé neutre 》 dans la consigne d'un exercice $\left(\mathrm{N}^{\circ} 5\right)$ : «ces énoncés comportent des marques de modalisation, identifiez-les puis réécrivez les phrases de manière neutre et objective " (Manuel scolaire de $3^{\text {ème }}$ A.S : 62).

Ce rapprochement du concept de «neutralité » avec celui d' «objectivité » pose problème :

Un énoncé "neutre" [...] se définit donc par une absence de prise de position du locuteur (ou de point de vue, dans le sens argumentatif du terme) ; un énoncé "objectif" quant à lui peut comporter des traces du point de vue pris en charge par le locuteur. (Boch et Gossmann, $2009: 3)$ 
Ainsi, dans l'optique de la modalisation, « neutralité » et " objectivité » réfèrent à des comportements différents, voire opposés ; leur rapprochement synonymique est donc erroné.

Cette observation est valable pour l'enseignement de la modalisation au niveau des discours direct et indirect.

Dans les nouveaux manuels du secondaire, leur présentation subit peu de modifications par rapport aux anciens manuels scolaires :

Le discours direct rapporte les propos prononcés sans aucune modification, ce n'est pas le narrateur qui parle mais l'un des personnages, les paroles rapportées au discours direct font référence à la situation d'énonciation du personnage et non celles du narrateur. Par contre le discours indirect fait partie intégrante du système d'énonciation du narrateur. (Manuel de

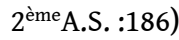

Alors que ce type de modalisation pourrait s'avérer essentiel pour marquer une prise de position d'un énonciateur, on ne retrouve, dans les manuels, que l'opposition classique entre discours direct et discours indirect, posée comme une évidence formelle. Ainsi, la plupart des exercices proposés restent des exercices de transformation d'un style à l'autre : «mettez les passages suivants au style direct » (manuel de $2^{\text {ème }}$ A.S. : 187). Leur conception, purement formelle, n'établit aucune relation effective entre le type de discours et la situation d'énonciation, d'autant que les énoncés sont pris isolément, hors de tout contexte.

$\mathrm{Au}$ terme de cette étude, nous pouvons inférer que la théorie sous-jacente à l'enseignement du français dans le cycle secondaire est la grammaire énonciative, ou grammaire notionnelle fonctionnelle, et que la progression grammaticale est en relation avec les actes de parole à acquérir dans chaque séquence.

Cette grammaire a intéressé les concepteurs de ces programmes parce qu'elle repose sur le principe selon lequel l'apprenant, au début de son apprentissage d'une langue étrangère, n'est pas motivé par le métadiscours grammatical (ou l'acquisition des notions grammaticales) mais par le lexique. On peut donc se contenter de lui inculquer peu de notions mais en situation de communication; d'où la place de la linguistique d'énonciation à un niveau pragmatique. Cependant, même à ce niveau, la didactisation de l'énonciation reste difficile à concevoir et les lacunes qu'elle présente, quel que soit le contexte, exigent l'intervention des spécialistes.

\section{BIBLIOGRAPHIE}

BALLY, Ch., "Syntaxe de la modalité explicite », Cahier F. de Saussure, 2, Genève, Cercle Ferdinand de Saussure, 1942, pp. 3-13.

BOCH, F., GROSSMANN, F., «L'énonciation dans les manuels scolaires de troisième », in Lidil, 35/2007, [en ligne], mis en ligne le $1^{\text {er }}$ décembre 2008. [Disponible sur URL :

< https://journals.openedition.org/lidil/2163 > 
COURTILLON J., « Pour une grammaire notionnelle », in Langue Française, Volume 68, $\mathrm{N}^{\circ} 1$, Larousse, 1985, pp. 32-47. Consultable sur le site :

< http://www.persee.fr/doc/lfr_00238368_1985_num_68_1_6353 >

ELALOUF, M. L., KERINEC, M., « Un groupement de textes sur la modalisation au lycée », in Le Français aujourd'hui, n 128, 1999, pp. 99-109.

KERBRAT ORECCHIONI, C., L'énonciation. De la subjectivité dans le langage, Paris, Armand Colin, 1988.

Manuel scolaire de $1^{\text {ère }}$ A.S, Office national des publications scolaires, 2005.

Manuel scolaire de $2^{\text {ème }}$ A.S, Office national des publications scolaires, 2005.

Manuel scolaire de $3^{\text {ème }}$ A.S, Office national des publications scolaires, 2005.

Programme de $1^{\text {ère }}$ A.S, janvier 2005. Consultable sur le site :

< http://www.oasisfle.com/documents/

nouveau_programme_de_francais_1ere_annee_secondaire.htm\#5_-_Le_cadre_théorique >

Programme de $2^{\text {ème }}$ A.S, septembre 2005.

Programme de $3^{\text {ème }}$ A.S, septembre 2006. Consultable sur le site :

< http://fle.ucoz.com/docdacc3emeas.doc >

\section{NOTES}

1. PARE : «Programme d'Appui de l'UNESCO à la Réforme du système Educatif : La Refonte de la pédagogie en Algérie. Défis et enjeux d'une société en mutation », 2005. Consultable sur le site :

< http://unesdoc.unesco.org/images/0014/001498/149828f.pdf >

2. Consultable sur le site : < http://fle.ucoz.com/docdacc3emeas.doc >

3. Consultable sur le site :

$<$

http://www.oasisfle.com/documents/ nouveau_programme_de_francais_1ere_annee_secondaire.htm\#5_-_Le_cadre_théorique >

4. Par exemple, à l'oral, "l'élève est capable de:-distinguer le texte argumentatif du texte narratif, descriptif ou explicatif, -reformuler un court énoncé narratif, descriptif ou explicatif, -produire un court énoncé narratif, descriptif, explicatif ou argumentatif, - étayer une argumentation à l'aide d'arguments, d'exemples et d'explications.». Le profil à l'écrit est sensiblement identique à celui de l'oral» (cf.

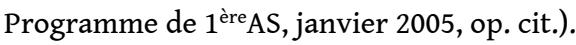

5. Cet «objet d'étude» est ainsi introduit dans le programme de $3^{\text {ème }}$ AS (septembre 2006, op. cit.) : "l'étude des textes argumentatifs en $3^{\text {ème }}$ année secondaire portera sur la mise en évidence des stratégies d'appel visant à faire agir ou réagir (l'exhortation), ainsi que celle des textes polémiques (le débat d'idées)».

6. "L'énonciation dans les manuels scolaires de 3ème", Lidil, Revue de linguistique et de didactique des langues, $\mathrm{N}^{\circ} 35,2007$, pp. 25-40. Mis en ligne le 1er décembre 2008. URL :

$<$ https://journals.openedition.org/lidil/2163 > 


\section{RÉSUMÉS}

Dans cet article nous avons tenté de cerner la difficulté de didactiser une notion linguistique telle que l'énonciation dans les manuels scolaires algériens. Nous nous appuyons sur un corpus d'étude qui comprend les programmes et les manuels scolaires de français du secondaire publiés dans le cadre de la réforme scolaire, pour faire émerger les théories d'énonciation qui soustendent leurs discours. Nous montrons également que les données de l'énonciation retenues s'y manifestent ponctuellement et en ordre dispersé dans leur application à l'explication de texte et à l'enseignement des points de langue.

In this article we have tried to highlight the difficulty of using, in a didactic context, a linguistic notion such as the enunciative one as illustrated in the Algerian secondary textbooks. For this purpose, we relied on a corpus of study that includes school curricula and textbooks used in teaching French at the secondary school as published within the framework of the school reform, to bring out the enunciation theories underlying their speech. Besides, we also show that the data of enunciation show punctually themselves and in a disorganized way in their application in the explanation of texts and language points.

INDEX

Keywords : grammar, educational grammar, enunciative, textbooks, programs

Mots-clés : grammaire, grammaire pédagogique, énonciation, manuels scolaires, programmes du secondaire

\section{AUTEUR}

AHLEM IDJET

ENS - Constantine Algérie 